\title{
Epidemiological profile and distribution of prognostic factors in invasive breast cancer among Algerian women
}

\author{
Amel Elbasyouni', Leila Saadi ${ }^{1,2}$, AbdelKarim Baha ${ }^{3}$ \\ ${ }^{1}$ Department of Biology, SNV Faculty, Blida 1 University, Algeria \\ ${ }^{2}$ Animal Ecobiology Laboratory, Higher Normal School, Kouba, Algiers, Algeria \\ ${ }^{3}$ Anatomy Pathology Service, CHU Beni Messous, Algiers, Algeria
}

Correspondence:

Amel Elbasyouni Department of Biology, SNV Faculty, Blida 1 University, Algeria e-mail:elbasyouni.amel@hotmail.com

Received:

29.07.2021

Accepted:

22.09.2021

DOI: 10.24292/01.OR.124220921

Copyright $\odot$ Medical Education. All rights reserved.

\section{ABSTRACT}

Although the widespread of early screening and advanced medical therapies, the breast cancer incidence rate continues to rise among Algerian women. This retrospective study investigated mammary lesions' epidemiological profile and histopathological characteristics and evaluated primary invasive breast cancer prognostic factors. We found that the incidence of breast cancer increases in middle-aged women between 40 and 60 years. Scarff Bloom Richardson grade II predominates in invasive breast cancer samples. In this study, molecular profiling shows that $82.1 \%$ of invasive tumours are hormone receptor-positive. A significant correlation is observed between the age of the patient and the SBR grade $(p=0.001)$ and with the hormone receptor expression $(p=0.001)$. In addition, the tumour grade is significantly correlated to oestrogen and progesterone receptor expression $(p=0.000$; $p=0.000$, respectively). Twenty-two per cent of cases were human epidermal growth factor receptor 2-positive. The Ki-67 proliferation index is expressed in $91 \%$ of breast cancer patients and was significantly associated with Scarff Bloom Richardson grade $(p=0.030)$, the progesterone receptor expression $(p=0.029)$ and with human epidermal growth factor receptor 2 -positivity $(p=0.023)$. Primary breast cancer with a high grade is more frequent (31\%) in young women under 40 years old, presenting $17 \%$ of our population. In summary, breast cancer patients in Algeria develop an unfavourable profile. Immunohistochemistry assay has played a pivotal role in assessing breast cancer predictive biomarkers improving the tumour behaviour and response to treatment.

Key words: breast cancer, epidemiology, biomarkers, immunohistochemistry, prognostic 


\section{INTRODUCTION}

The incidence of breast cancer in the world and Algeria has grown steadily and continues to increase in recent years [1, 2]. In 2018, more than 2.1 million women worldwide were diagnosed with breast cancer, and about 627,000 death were recorded [3, 4]. Algerian women are more affected by breast carcinoma than other types of cancer [5]. Mammary carcinoma has a high heterogeneity [6] concerning histology [7], molecular modification characteristics, alteration specificity [8, 9]. Several factors such as histological grade, tumour histological type, oestrogen receptor (ER) and human epidermal growth factor receptor-2 (HER2/neu) affect cancer prognostics and response to therapy. Regarding molecular subtyping [10], classical immunohistochemistry (IHC) of breast cancer markers, including oestrogen receptor (ER), progesterone receptor (PR) and HER2, become simpler, more cost-effective and play a critical role [11]. Hence, the prognosis is highly related to cancer malignancy and progression [12].

To our knowledge, the main challenge is to identify the good therapy option adapted to each patient individually [13]. For this reason, we conducted this study to explore the epidemiological profile of invasive breast cancer and patterns of expression of prognostic factors among Algerian women to improve our awareness and contribute to better management of health care among patients.

\section{MATERIAL AND METHODS}

This retrospective study was for the benefit of women with breast cancer, collected at the anatomopathology laboratory at the Isaad Hassani University Hospital in Beni-Messous, Algiers. We analysed the histologic reports of 1037 mammary biopsies' microscopic examination assessed between January 2011 and December 2019. For all cases, pathologists have performed histology examination to identify the histopathological aspect according to the world health organization classification and graded according to Nottingham modification of Scarff Bloom Richardson (SBR) grading system [14, 8]. Pathologists undergo an immunohistochemical staining examination for all confirmed breast cancer cases to evaluate prognostic markers' expression that may help oncologists decide an effective therapeutic procedure. The immunohistochemical panel include oestrogen receptors, progesterone receptor, human epidermal growth factor type 2 receptor and Ki-67. Labelling the expression of the hormonal receptor is based on the Quick score according to Allred 2010 [15]. The analysis leads to determining a score resulting from the percentage of positive carcinomatous cells and marking inten- sity. A score of $(+2)$ or lower reflects negativity, and $(+8)$ is the maximum score that could be obtained.

In contrast, we considered only membrane labelling while the evaluation of HER2. The analysis must specify the proportion of labelled cells and labelling intensity with scores ranging from (0) to $(+3)$. Only a (+3) score is considered positive [16]. We considered only nuclear labelling in immunohistochemistry assays.

In our study, data were analysed statistically using IBM SPSS statistics 25 software. The descriptive study revolves on the mean, standard deviation and percentage of the collected data that articulates age, gender, and nature of the mammary lesion, histological type, Scarff Bloom Richardson (SBR) grade and ER, PR, HER2 and Ki-67 immunohistochemical results. We performed a Chi2 test to check the correlation between the different studied prognostic parameters.

The results were statistically considered significant at a p-value $<0.05$, which indicates strong evidence against the null hypothesis, as there is less than a $5 \%$ probability the null is correct.

\section{RESULTS}

In our study, we reported 1023 women diagnosed with breast pathology. Among them, 455 (44\%) were histologically confirmed as breast cancer cases, against 568 (56\%) cases were breast benign diseases. Only 14 cases occurred in men - seven cases $(0.68 \%)$ are benign lesions, the other seven cases $(0.68 \%)$ were breast cancer cases - and we excluded them from further analysis in our study.

Four hundred thirty-five (435) reports that provide age; we found that the mean age of our breast cancer patients is $50.29 \pm 12.24$ with extremes ranging from 21 to 101 years old with a pic of incidence between 50 and 53 years old and decrease after the 60 s. Table 1 shows the distribution of our patients in the three age groups. Histologically, infiltrating ductal carcinoma was mostly found in 389 (85\%) of patients, and only 61 (15\%) were diagnosed by an in situ carcinoma. In the cohort, the rank of SBR grade was evaluated in 355 (78\%) sample, in which grade II is the most frequent that occurred in 244 (69\%) case, followed by 84 (24\%) of grade III. Only 27 (7\%) cases were of grade I. We found a statistically significant difference between the distributions of the three modalities of SBR grade in the different age groups, as shown in table 1. 
Table 1. Distribution of invasive breast cancer cases according to SBR grade and hormone receptor expression in the three age groups.

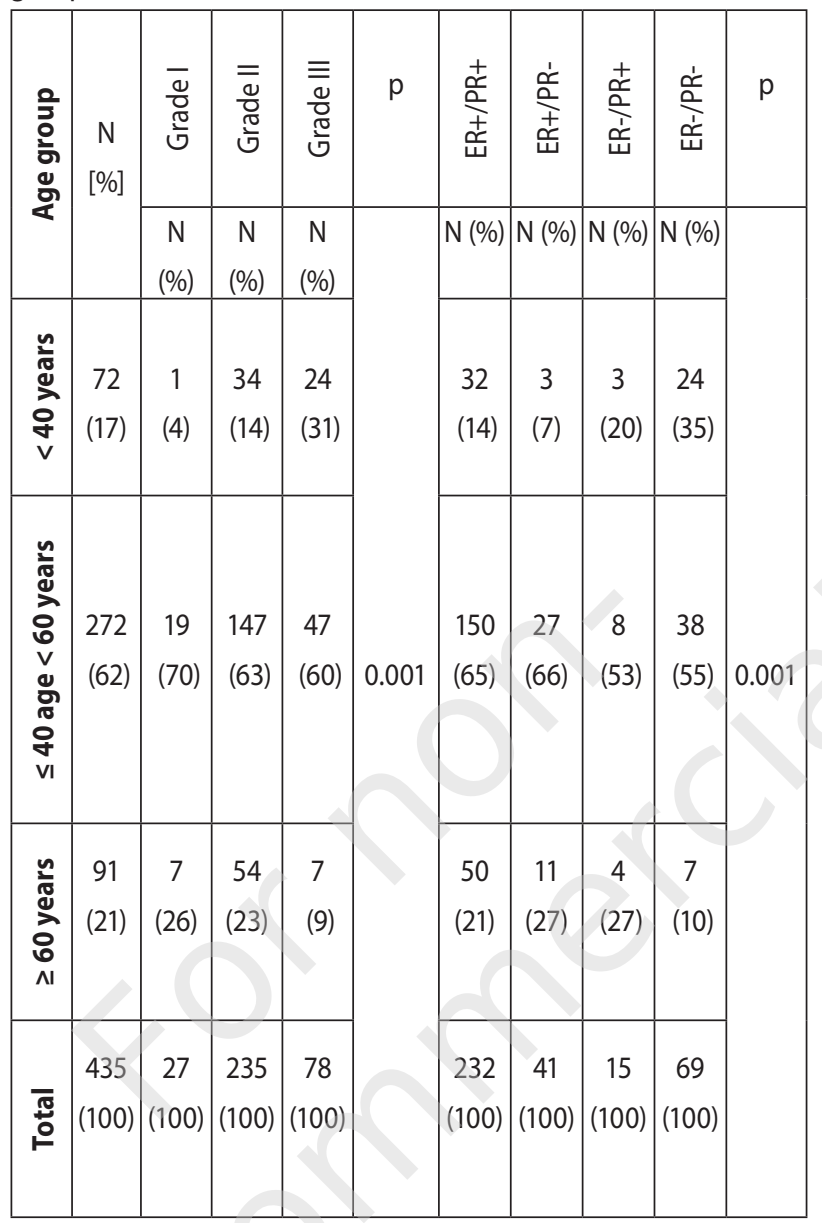

\section{IMMUNOHISTOCHEMICAL EVALUATION OF ER, PR AND HER2 IN INVASIVE BREAST CARCINOMA CASES}

In this study, the immunohistochemical staining of hormone receptors was performed in 402 (88\%) cases. Hormones receptors are co-expressed $\left(\mathrm{ER}^{+} / \mathrm{PR}^{+}\right)$in $233(58 \%)$ samples. The oestrogen receptor is individually expressed in 80 (19.9\%) samples, and for that, $77.9 \%$ of tumours are oestrogen receptor-positive. Progesterone receptor is marked in $62.2 \%$ of samples, and it is individually expressed in 17 (4.2\%) tumours. While most cases are hormone-dependent growth patterns, 72 (17.9\%) show a negative hormone receptor expression (ER/PR).

We noticed that in young women, tumours tend to lose hormone receptors. Middle-aged women evenly expressed the four phenotypes of hormone receptors. In the current study, we found a significant correlation between hormone receptor expression and the age of patients at the moment of diagnosis, as shown in table 1.
Regarding the differentiation of mammary parenchyma according to hormones receptors status, the ER was expressed strongly in grade I (93\%) and grade II (85\%) tumours. This rate has decreased to $41 \%$ in grade III tumours. However, in tumours that lack oestrogen receptor, the frequency of grade III was greater $(59 \%)$ than that of grade II (15\%) and grade I (7\%) tumours. In independent-hormone breast cancer cases, we noticed that the frequency of grade III was higher than that of grade II or grade I, as shown in table 2.

Table 2. Distribution of invasive breast cancer cases according to oestrogen and progesterone receptor status and SBR grade.

\begin{tabular}{|c|c|c|c|c|}
\hline \multirow{2}{*}{ Phenotype } & Grade I & Grade II & Grade III & \multirow{2}{*}{$\mathrm{p}$} \\
\cline { 2 - 4 } & $\mathrm{N}(\%)$ & $\mathrm{N}(\%)$ & $\mathrm{N}(\%)$ & \\
\hline ER+ & $25(93)$ & $204(85)$ & $34(41)$ & \multirow{2}{*}{0.000} \\
\hline ER- & $2(7)$ & $37(15)$ & $48(59)$ & \\
\hline Total & $27(100)$ & $241(100)$ & $82(100)$ & \\
\hline PR+ & $25(93)$ & $178(74)$ & $38(46)$ & \multirow{2}{*}{0.000} \\
\hline PR- & $2(7)$ & $63(26)$ & $45(54)$ & \\
\hline Total & $27(100)$ & $241(100)$ & $83(100)$ & \\
\hline
\end{tabular}

HER2 overexpression was recorded in 81 (22\%) cases, while the majority of tumours (61\%) lack expression of this oncogene and show a score of $(0)$ or $(+1)$. Sixty-four (17\%) cases show an equivoque expression of HER2 (score +2$)$. In this case, the gene amplification test using fisher in situ hybridization technique (FISH) is recommended to justify whether the tumour is HER2-positive or negative. Unfortunately, this technique is not available in our laboratory.

\section{KI-67 PROLIFERATION INDEX EXPRESSION AND ITS ASSOCIATION WITH OTHER PROGNOSTIC FACTORS IN INVASIVE BREAST CANCER CASES}

Among our patients, the $\mathrm{Ki}-67$ is positive in $90.6 \%$ (310 of 342 ) of tumours and negative in only 32 tumours (9.4\%). Tumours with high proliferation activity are mostly positive for oestrogen receptor (76\%) and progesterone receptor (69\%). However, there is no significant correlation between $\mathrm{Ki}-67$ expression and age $(p=0.406)$, nor with oestrogen receptor expression $(p=0.135)$. Nevertheless, we found a significant correlation between $\mathrm{Ki}-76$ and grade $(p=0.030)$, the expression of the progesterone $(p=0.029)$ and epidermal growth factor HER2 $(p=0.023)$ among patients in this cohort (tab. 3). 
Table 3. Distribution of invasive breast cancer cases according to Ki-67 expression status in correlation with histological grade, progesterone and Human epidermal growth factor 2 receptor state.

\begin{tabular}{|c|c|c|c|c|}
\hline \multirow{2}{*}{\multicolumn{2}{|c|}{ Characteristics }} & Ki-67 - & Ki-67+ & \multirow{2}{*}{ p-value } \\
\hline & & $\mathbf{N}(\%)$ & N (\%) & \\
\hline \multirow{4}{*}{ Grade } & I & $5(17)$ & $18(6)$ & \multirow{3}{*}{0.030} \\
\hline & II & $22(73)$ & $205(69)$ & \\
\hline & III & $3(10)$ & $74(25)$ & \\
\hline & Total & $30(100)$ & $297(100)$ & \\
\hline \multirow{3}{*}{ PR } & Negative & $4(12.5)$ & $96(31)$ & \multirow{2}{*}{0.029} \\
\hline & Positive & $28(87.5)$ & $214(69)$ & \\
\hline & Total & $32(100)$ & $310(100)$ & \\
\hline \multirow{4}{*}{ HER2 } & $\begin{array}{l}\text { Negative } \\
(0 \text { or }+1)\end{array}$ & $25(78)$ & $177(58)$ & \multirow{3}{*}{0.023} \\
\hline & $\begin{array}{c}\text { Equivoque } \\
\qquad(+2)\end{array}$ & $6(19)$ & $56(18)$ & \\
\hline & $\begin{array}{l}\text { Positive } \\
\qquad(+3)\end{array}$ & 1 (3) & $72(24)$ & \\
\hline & Total & $32(100)$ & $305(100)$ & \\
\hline
\end{tabular}

\section{DISCUSSION}

Regarding the heterogeneity of breast cancer $[17,18]$, the latest classifications established by the World Health Organization take into account the variability of the morphological, phenotypic and molecular profile of this type of cancer [6,9]. The state of hormone receptors has become a predictor of response to hormonal therapy with tamoxifen in women with hormone-dependent breast cancer [19]. Expression of the progesterone receptor is crucial in cancerous mammary parenchyma. Once active, it triggers cell proliferation. Therefore, the progesterone receptor is a predictor of the therapeutic response; however, its labelling is not essential for prognosis [20,21].

In the present study, the majority of breast cancer cases were hormone-dependent. A recent study conducted in India shows that $42.8 \%$ were oestrogen receptor-positive tumours and $31.8 \%$ were PR-positive [22]. Clearly, oestrogen is a carcinogen hormone. The exposition to that ovarian steroid hormone lead the accumulation of DNA damage, aneuploidy, chromosome's loss and gain and a hyperméthylation of tumour suppression genes $[23,24]$. In addition, exposition to endocrine disruptors may interact with oestrogen receptors and lead to an abnormal epigenetic profile and nuclear instability [25].

Although ER, a driving transcription factor in oestrogen-dependent breast cancer, other receptors may affect tumour progression by modulating ER function - most notably the PR. This steroid receptor is expressed in more than $75 \%$ of $\mathrm{ER}^{+}$breast cancer cases and reflects to active ER pathway. Therefore PR expression is a predictive biomarker of patient outcome $[26,27]$. Increasingly, PR plays a direct functional role in controlling tumour progression. Indeed, progestogens are efficient to inhibit breast tumour growth [28]. The mechanism involves PR-directed ER distribution to PR-binding sites by sequestering ER before the activation/ expression of a pro-proliferative target gene, thereby inhibiting tumour genesis or progression [28]. This mechanism could be highly effective in tamoxifen-resistant breast cancer cells.

Among our patients, we noted a significant correlation between hormone receptor expression and the age of patients. The literature shows a very good correlation between the age of diagnosis and oestrogen receptor expression [29]. In another study, the frequency of tumours expressing hormonal receptors increases after the 40s ( $p=0.03$ ) [30]. Nevertheless, ER/PR phenotype is more frequent in patients under 50 years (68.1\%), and the $E R^{+} / \mathrm{PR}^{+}$phenotype is more present in patients aged 50 and over [31].

Our patients developed more frequently a tumour of SBR grade II. Other studies confirm this finding [31, 32]. The histological grade is the most related to the hormone receptors content in the tumour. As the tumour becomes more anaplastic, we observe a uniform loss of ER content, indicating that hormone receptor status may represent an aspect of differentiation of parenchymal and tumour cells. The loss of oestrogen receptors are associated with an inadequate response to endocrine therapy and show a worse prognosis [33, 34].

We reported a significant correlation between SBR grade and both hormonal receptors individually. An inverse association was reported between hormone receptor expression and the histologic grade, a $p$-value $<0.001$ [35].

Moreover, overexpression of HER2 oncogene occurs in $15-20 \%$ of patients with mammary carcinoma, leading to an aberrant constitutive activation of its signalling pathway and favouring uncontrolled cell growth [19,36-38]. Thus, HER2 is a prognostic and a predictive marker to trastuzumab and lapatinib drugs responses $[38,39]$. In the current study, $22 \%$ of tumours overexpress this oncogene. The obtained results agree with those found in other 
research. $14.4 \%$ of cases in Peru highlighted an overexpressed HER2 [40] and among Egyptian women in $19.6 \%$ of cases [41]. However, this frequency was higher in some other studies and varied over the years. Our results were lower than those reported in Iran (44.5\%) [42]. In 2017, 40.7\% of cases were HER2 positive in India and 43.2\% in 2010 [22, 43]. In western Algeria, the number of tumours was higher in 2014 , with $47.50 \%$ out of a population of 240 patients [44]. The variation in the expression of HER2 is based on ethnicity and age at diagnosis.

Expression of Ki-67 in patients with invasive mammary carcinoma was observed mainly in premenopausal women due to the proliferative and anti-apoptotic effect linked to the high expression of hormone receptors during this age period [45]. We found a significant correlation of Ki-67 expression with SBR grade, HER2 and progesterone receptor expression. Our finding agrees with other studies [46-49].

The introduction of Ki-67 labelling in the immunohistochemistry staining panel as a diagnostic tool is important in planning adjuvant therapy, especially for administering additional chemotherapy in hormone-dependent breast cancer patients [50].

Kurbel et al. (2017) suggested that the expression of PR in breast cancer cells is related to the value of Ki-67 [49]. They noted that the lack of androgen receptors in $\mathrm{ER}^{+}$tumours is associated with a poor prognosis. While Elkablawy et al. (2016) showed that Ki-67 expression is significantly associated with poor prognostic criteria, including advanced age $(p<0.02)$, high tumour grade $(p<0.01)$ and HER2 expression $(p<0.009)$ [50]. However, Kermani et al. (2019) found that Ki-67 expression was significantly correlated with oestrogen and progesterone receptor expression but not with age or tumour grade. In another study carried out in Japan on 3652 patients [47], the researchers revealed that the overexpression of $\mathrm{Ki}-67$ in breast carcinomas was significantly correlated with a high SBR grade (grade III, poorly differentiated tumour; $p<0.0001$ ). Nishimura et al. (2010) have also reported a significant correlation between $\mathrm{Ki}-67$ expression and age $(p=0.0001)$, and its frequency was greater in women aged $>50$ years than in young women under 30 years [46]. Chen et al. (2016) [51, 52] and Varga et al. (2019) [48] confirmed the significance of Ki-67 as an effective diagnostic tool for planning adjuvant therapy, particularly for the treatment of patients with hormone-sensitive breast cancer.

Breast cancer presents inter- and intra-tumour heterogeneity. This heterogeneity results from the combination of different variables: the cellular origin, genetic and epigenetic changes and the environmental context [53]. In addition, the factors influencing the risk of developing breast cancer are linked to the molecular profiles of tumours. They affect the biology and clinical behaviour of tumours that occur later. Consequently, the molecular profiles of mammary carcinomas are fixed from the onset of the tumour process [54].

\section{CONCLUSIONS}

According to our findings, Algerian women with breast cancer show unfavourable outcomes. While the anatomic-histopathological and immunohistochemical criteria of breast cancer samples are crucial at diagnosis, the prognosis of breast cancer is closely related to the progression of the disease. The earlier the diagnosis, the better the survival. In Algeria, the cancer screening organization is one of the main points of the 2015-2019 cancer plan. At present, the treatment and care of breast cancer patients have reached a high standard, and the major challenge lies in distinguishing which tumours need to be treated more aggressively and identifying the best therapeutic option adapted to each patient. This goal could not be achieved unless if the information clarifying the biology of tumour is transferred completely and successfully to the clinical axe.

\section{References}

1. Benarba B, Meddah B, Hamdani H. Cancer incidence in North West Algeria (Mascara) 2000-2010: results from a population-based cancer registry. EXCLI Journal. 2014; 15

2. Azamjah N, Soltan-Zadeh Y, Zayeri F. Global Trend of Breast Cancer Mortality Rate: A 25-Year Study. Asian Pac J Cancer Prev. 2019; 20(7): 2015-20.

3. Mei J, Zhao J, Fu Y. Molecular classification of breast cancer using the mRNA expression profiles of immune-related genes. Sci Rep. 2020; 10(1): 4800.

4. Negro G, Aschenbrenner B, Brezar SK et al. Molecular heterogeneity in breast carcinoma cells with increased invasive capacities. Radiol Oncol. 2020 54(1): 103-18.

5. Tfaily MA, Nassar F, Sellam L-S et al. Calin G (ed). miRNA expression in advanced Algerian breast cancer tissues. PLoS ONE. 2020; 15(2): e0227928.

6. Turashvili G, Brogi E. Tumor Heterogeneity in Breast Cancer. Front Med. 2017; 4: 227.

7. Makki J. Diversity of Breast Carcinoma: Histological Subtypes and Clinical Relevance. Clin Med Insights Pathol. 2015; 8: 23-31. http://doi.org/10.4137/ CPath.S31563.

8. Harbeck N, Penault-Llorca F, Cortes J et al. Breast cancer. Nat Rev Dis Primers. 2019; 5(1): 66.

9. Tsang JYS, Tse GM. Molecular Classification of Breast Cancer. Adv Anat Pathol. 2019; 27(1): 9.

10. Viale G. The current state of breast cancer classification. Ann Oncol. 2012; 23: x207-10. 
11. Goldhirsch A, Wood WC, Coates AS et al. Strategies for subtypes - dealing with the diversity of breast cancer: highlights of the St Gallen International Expert Consensus on the Primary Therapy of Early Breast Cancer 2011. Ann Oncol. 2011; 22(8): 1736-47.

12. Shah T, Guraya S. Breast cancer screening programs: Review of merits, demerits, and recent recommendations practiced across the world. J Microsc Ultrastruct. 2017; 5(2): 59.

13. Testa U, Castelli G, Pelosi E. Breast Cancer: A Molecularly Heterogenous Disease Needing Subtype-Specific Treatments. Med Sci. $2020 ; 8(1): 18$.

14. Sinn HP, Kreipe H. A Brief Overview of the WHO Classification of Breast Tumors, 4th Edition, Focusing on Issues and Updates from the 3rd Edition. Breast Care. 2013; 8(2): 149-54.

15. Qureshi A, Pervez S. Allred scoring for ER reporting and it's impact in clearly distinguishing ER negative from ER positive breast cancers. J Pak Med Assoc. 2010; 60(5): 5.

16. Marchiò C, Annaratone L, Marques A et al. Evolving concepts in HER2 evaluation in breast cancer: Heterogeneity, HER2-low carcinomas and beyond. Seminars in Cancer Biology. 2020; S1044579X20300493.

17. Vincent-Salomon A. Classification morphologique des carcinomes mammaires de type rare. Lett Cancérol. 2013; XXII(4).

18. Atif N. Role of immunohistochemical markers in breast cancer and their correlation with grade of tumour, our experience. ICPJL [Internet]. 2018; 6(3). https://medcraveonline.com/ICPL/role-of-immunohistochemical-markers-in-breast-cancer-and-their-correlation-with-grade-of-tumour-our-experience.html (access: 13.06.2020).

19. Barzaman K, Karami J, Zarei Z et al. Breast cancer: Biology, biomarkers, and treatments. Int Immunopharmacol. $2020 ; 84: 106535$.

20. Gradishar WJ, Anderson BO, Balassanian R et al. Breast Cancer Version 2.2015. J Natl Compr Canc Netw. 2015; 13(4): 28.

21. Guiu S, Charon-Barra C, Vernerey D et al. Coexpression of androgen receptor and FOXA1 in nonmetastatic triple-negative breast cancer: ancillary study from PACS08 trial. Future Oncol. 2015; 11(16): 2283-97.

22. Bansal C, Sharma A, Pujani M et al. Correlation of hormone receptor and human epidermal growth factor Receptor-2/neu expression in breast cancer with various clinicopathologic factors. Indian J Med Paediatr Oncol. 2017; 38(4): 483.

23. Danforth DN. Molecular profile of atypical hyperplasia of the breast. Breast Cancer Res Treat. 2018; 167(1): 9-29.

24. Vogel VG. Epidemiology of Breast Cancer. In: The Breast [Internet]. Elsevier; 2018: 207-18.e4. https://linkinghub.elsevier.com/retrieve/pii/ B9780323359559000155 (access: 4.07.2020).

25. Mufudza C, Sorofa W, Chiyaka ET. Assessing the Effects of Estrogen on the Dynamics of Breast Cancer. Comput Math Methods Med. 2012; 2012: 1-14.

26. Blows FM, Driver KE, Schmidt MK et al. Subtyping of Breast Cancer by Immunohistochemistry to Investigate a Relationship between Subtype and Short and Long Term Survival: A Collaborative Analysis of Data for 10,159 Cases from 12 Studies. PLoS Med. 2010; 7(5): e1000279.

27. Purdie CA, Quinlan P, Jordan LB et al. Progesterone receptor expression is an independent prognostic variable in early breast cancer: a population-based study. Br J Cancer. 2014; 110(3): 565-72.

28. Carroll JS, Hickey TE, Tarulli GA et al. Deciphering the divergent roles of progestogens in breast cancer. Nat Rev Cancer. 2017; 17(1): 54-64.

29. McGuire A, Brown J, Malone C et al. Effects of Age on the Detection and Management of Breast Cancer. Cancers. 2015; 7(2): 908-29.

30. AlZaman A, Mughal S, AlZaman Y et al. Correlation between hormone receptor status and age, and its prognostic implications in breast cancer patients in Bahrain. SMJ. 2016; 37(1): 37-42.

31. Al-Nuaimy WMT, Ahmed AH, Al-Nuaimy HAA. Immunohistochemical Evaluation of Triple Markers (ER, PR and HER-2/neu) in Carcinoma of the Breast in the North of Iraq. DJMLD. 2015; 1(1): 001-9.

32. Sofi GN, Sofi JN, Nadeem R et al. Estrogen Receptor and Progesterone Receptor Status in Breast Cancer in Relation to Age, Histological Grade, Size of Lesion and Lymph Node Involvement. Asian Pac J Cancer Prev. 2012; 13(10): 5047-52.

33. Kuukasjärvi T, Kononen J, Helin $\mathrm{H}$ et al. Loss of estrogen receptor in recurrent breast cancer is associated with poor response to endocrine therapy. JCO. 1996; 14(9): 2584-9

34. Saedi HS, Nasiri M-RG, ShahidSales S et al. Comparison of Hormone Receptor Status in Primary and Recurrent Breast Cancer. Iran J Cancer Prev. 2012; 5(2): 5 .

35. Mahmoud MM, Mahmoud M. Breast Cancer in Kirkuk City, Hormone Receptors Status (Estrogen and Progesterone) and Her-2/Neu and Their Correlation with Other Pathologic Prognostic Variables. J Med. 2014; 6(1): 14

36. Furrer D, Paquet C, Jacob S et al. The Human Epidermal Growth Factor Receptor 2 (HER2) as a Prognostic and Predictive Biomarker: Molecular Insights into HER2 Activation and Diagnostic Implications. In: Lemamy GJ (ed). Cancer Prognosis [Internet]. IntechOpen. 2018. https://www.intechopen. com/books/cancer-prognosis/the-human-epidermal-growth-factor-receptor-2-her2-as-a-prognostic-and-predictive-biomarker-molecular (access: 10.06.2020).

37. Hsu JL, Hung MC. The role of HER2, EGFR, and other receptor tyrosine kinases in breast cancer. Cancer Metastasis Rev. 2016; 35(4): 575-88.

38. Cesca MG, Vian L, Cristóvão-Ferreira S et al. HER2-positive advanced breast cancer treatment in 2020. Cancer Treat Rev. 2020; 88: 102033.

39. Gaibar M, Beltrán L, Romero-Lorca A et al. Somatic Mutations in HER2 and Implications for Current Treatment Paradigms in HER2-Positive Breast Cancer. J Oncol. 2020; 2020: 1-13.

40. Yábar A, Meléndez R, Muñoz S et al. Effect of Ki-67 assessment in the distribution of breast cancer subtypes: Evaluation in a cohort of Latin American patients. Mol Clin Oncol. 2017; 6(4): 503-9.

41. Nahed AS, Shaimaa MY. Ki-67 as a prognostic marker according to breast cancer molecular subtype. Cancer Biol Med. 2016; 13(4): 496.

42. Keyhani E, Muhammadnejad A, Karimlou M. Prevalence of HER-2-Positive Invasive Breast Cancer: A Systematic Review from Iran. Asian Pac J Cancer Prev. 2012; 13(11): 5477-82.

43. Somasundaram K, Mukherjee G, Vaidyanathan K et al. ErbB-2 expression and its association with other biological parameters of breast cancer among Indian women. Indian J Cancer. 2010; 47(1): 8

44. Guedouar Y, Bekkouche Z, Ben Ali F et al. Évaluation phénotypique des sous-types moléculaires en carcinologie mammaire dans une population de I'Ouest algérien. J Afr Cancer [Internet]. 2014. http://link.springer.com/10.1007/s12558-014-0318-1 (access: 13.06.2020)

45. Mansouri H, Mnango LF, Magorosa EP et al. Ki-67, p53 and BCL-2 Expressions and their Association with Clinical Histopathology of Breast Cancer among Women in Tanzania. Sci Rep. 2019; 9(1): 9918.

46. Nishimura R, Osako T, Okumura $\mathrm{Y}$ et al. Ki-67 as a prognostic marker according to breast cancer subtype and a predictor of recurrence time in primary breast cancer. Exp Ther Med. 2010; 1(5): 747-54.

47. Kermani TA, Kermani IA, Faham Z et al. Ki-67 status in patients with primary breast cancer and its relationship with other prognostic factors. Biomed Res Ther. 2019; 6(2): 2986-91.

48. Varga Z, Li Q, Jochum W et al. Ki-67 assessment in early breast cancer: SAKK28/12 validation study on the IBCSG VIII and IBCSG IX cohort. Sci Rep. 2019; 9(1): 13534.

49. Kurbel S, Dmitrović $B$, Marjanović K et al. Distribution of Ki- 67 values within HER2 \& ER/PgR expression variants of ductal breast cancers as a potential link between IHC features and breast cancer biology. BMC Cancer. 2017; 17(1): 231.

50. Elkablawy M, Albasri A, Mohammed R et al. Ki67 expression in breast cancer. Correlation with prognostic markers and clinicopathological parameters in Saudi patients. SMJ. 2016; 37(2): 137-41.

51. Chen H, Zhou M, Tian W et al. Coleman WB (ed). Effect of Age on Breast Cancer Patient Prognoses: A Population-Based Study Using the SEER 18 Database. PLoS ONE. 2016; 11(10): e0165409. 
52. Chen G, Wu D, Guo W et al. Clinical and immunological features of severe and moderate coronavirus disease 2019. J Clin Invest. 2020; 130(5): 2620-9.

53. Anstine LJ, Keri R. A new view of the mammary epithelial hierarchy and its implications for breast cancer initiation and metastasis. JCMT [Internet]. 2019; 2019. https://jcmtjournal.com/article/view/3106 (access: 1.05.2020).

54. Yang Z, Yang M, Yu W et al. Molecular mechanisms of estrogen receptor $\beta$-induced apoptosis and autophagy in tumors: implication for treating osteosarcoma. J Int Med Res. 2019; 47(10): 4644-55.

Authors' contributions:

All authors contributed significantly to the study's conceptualization and design, data collection, analysis, and interpretation, writing and revising the paper, and final approval of the submitted version.

\section{Conflict of interests:}

None.

Financial support:

This research has not received any specific grant from public funding agencies, commercial or non-profit.

Ethics:

The authors had full access to the data and take full responsibility for its

integrity.

All authors have read and agreed with the content of the manuscript as

written

The paper complies with the Helsinki Declaration, EU Directives and harmonized requirements for biomedical journals. 